\title{
Green's Formulation for Chirowaveguides
}

\author{
M.J. Núñez-Trigueros, \\ G.J. Molina-Cuberos and J. Margineda \\ Electromagnetism and Electronics Dept., \\ University of Murcia, \\ E30100 Murcia, Spain
}

\author{
A.J. García-Collado \\ Polytechnic Sciences Dept., \\ Catholic University of San Antonio, \\ E30107 Murcia, Spain
}

\author{
Álvaro Gómez \\ Communication Engineering Department, \\ University of Cantabria, \\ E39005 Santander, Spain.
}

\begin{abstract}
A Green integral formulation of the boundary value problem in chirowaveguides with translational symmetry and the application to the rectangular case are presented in this paper. The Green equations for the two eigenmodes in unbounded chiral media, i.e. right and left circularly polarized waves, are formulated in terms of the Hankel functions. By splitting the waveguide contour into a finite number of intervals, the equations are discretized and a homogeneous system of equations can be obtained. The number of unknowns is reduced to the half by applying the relations between the longitudinal components and their normal derivatives at the metallic contours. The method has been used for modeling the rectangular waveguide and the dispersion diagram and the field structure for some propagation modes are presented.
\end{abstract}

\section{INTRODUCTION}

During the last decades, a high variety of new artificial materials, knowns as metamaterials, with bizarre properties have been designed and built. The new properties have challenged the capabilities of both theoretical and numerical techniques, which has triggered the development of new algorithms and methods [1], [2], [3]. Among those new materials, chiral media have attracted considerable attention and many theoretical and experimental papers on the interaction of electromagnetic waves in different waveguide structures have been published [4], [5], [6], [7]. Chiral media produce two main effects on a linearly polarized propagating wave: rotation of polarization angle, known as electromagnetic rotatory dispersion, and changes in the polarization from linear to elliptical, known as circular dichroism. It has been also found that strong electromagnetic activity can also produce negative refraction [8].

The scope of this paper is to model wave propagation in metallic waveguides with translational symmetry filled with chiral media (also known as chirowaveguides). By discretizing the Green integral equation for the two propagation modes in unbounded chiral media a numerical solution of the electromagnetic field inside the guide can be obtained. A similar method was used to solve the dielectric waveguide with PEC (perfect electric conductor) boundaries in [9], where a comparison with the analytical solution for rectangular and isosceles right triangular waveguides was also presented. Our model is first tested by solving the dielectric rectangular guide with PEC walls and by comparing with the known solution. Then the effects due to the chirality for several propagation modes will be discussed. This method is able to be adapted to most of the standard waveguide systems and also to open chirowaveguides.

\section{INTEGRAL EQUATIONS FOR CHIROWAVEGUIDES}

The Green's second identity in two dimensions for an uniform waveguide with arbitrary cross section $S$ and boundary $L$ is:

$$
\int_{S}\left[G \nabla_{t}^{2} \Phi-\Phi \nabla_{t}^{2} G\right] d s=\oint_{L}\left[G \partial_{n} \Phi-\Phi \partial_{n} G\right] d \ell,
$$

where $\vec{\nabla}_{t}=\hat{x} \partial / \partial x+\hat{y} \partial / \partial y, \partial_{n}=\hat{n} \cdot \vec{\nabla}_{t}$ and both the Green function $\left(G=G\left(\vec{r}_{0}, \vec{r}\right)\right)$ and $\Phi=\Phi(\vec{r})$ satisfy:

$$
\begin{aligned}
& \left(\nabla_{t}^{2}+\gamma^{2}\right) \Phi(\vec{r})=0, \\
& \left(\nabla_{t}^{2}+\gamma^{2}\right) G\left(\vec{r}_{0}, \vec{r}\right)=-\delta\left(\vec{r}_{0}-\vec{r}\right),
\end{aligned}
$$

being $\gamma$ a constant, $\delta\left(\vec{r}_{0}-\vec{r}\right)$ the Dirac function.

The application to an uniform dielectric waveguides with translational symmetry is straightforward by considering that each electromagnetic field component can be expressed as $\Phi(\vec{r}) e^{j(\omega t-\beta z)}$, where $\gamma^{2}=\omega^{2}-\beta^{2}$.

For the chiral media case, the presence of the chirality parameter, $\kappa$ in the constitutive equations

$$
\begin{aligned}
\vec{D} & =\epsilon \vec{E}-j \kappa \sqrt{\epsilon_{0} \mu_{0}} \vec{H}, \\
\vec{B} & =\mu \vec{H}+j \kappa \sqrt{\epsilon_{0} \mu_{0}} \vec{E},
\end{aligned}
$$

leads to a coupling in the electric and magnetic fields. This coupling can be overcome by decomposing the fields into the circularly polarized wavefield components [10] :

$$
\vec{E}^{ \pm}=(\vec{E} \mp j \eta \vec{H})
$$

where $\eta=\sqrt{\mu / \epsilon}$ is the wave impedance.

By eliminating the transverse field, two separated Helmholtz equations for both longitudinal wavefield components can be obtained [10]. The boundary conditions between metallic walls and chiral material must be also expressed in terms of the wave field components. By making use of the PEC boundary conditions in (1), integral expressions for $E_{z}^{+}(\vec{r})$ and $E_{z}^{-}(\vec{r})$ are obtained and their solutions provide the longitudinal component of the wavefields and its normal derivative. From $E_{z}^{ \pm}(\vec{r})$ and $\partial_{n} E_{z}^{ \pm}(\vec{r})$ the transversal components of the wavefields and, therefore, the whole electromagnetic field can be calculated. 

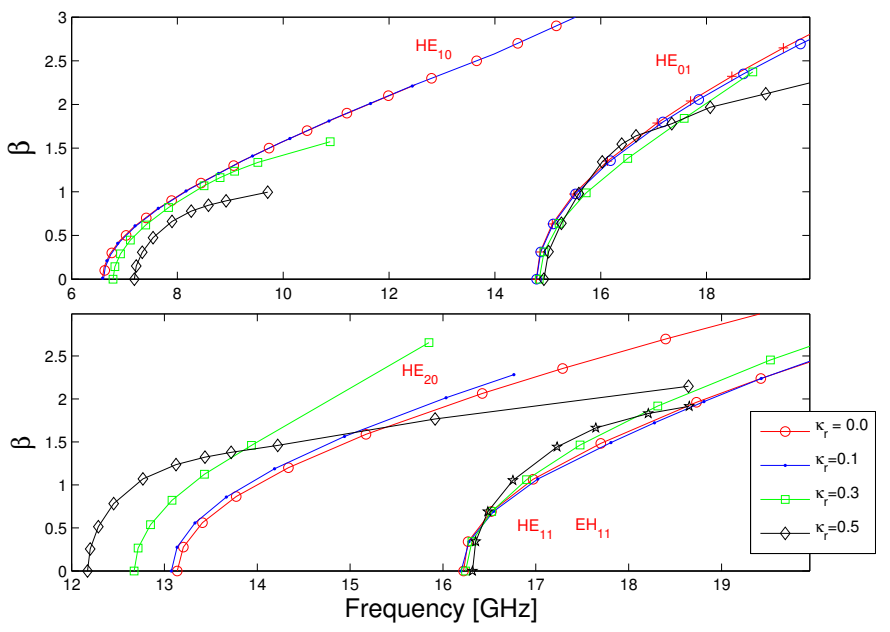

Fig. 1: Dispersion diagram of the rectangular chirowaveguide as a function of $\kappa_{r}$ with $\epsilon_{r}=1, \mu_{r}=1$.
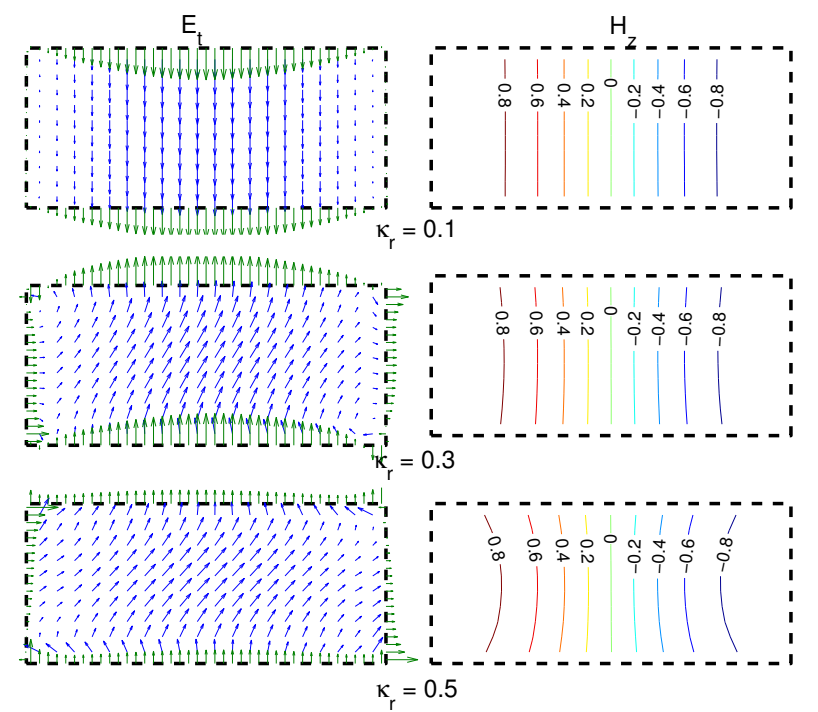

Fig. 2: $E_{t}$ and $H_{z}$ for the first propagation mode, $\mathrm{HE}_{10}$, and for $\kappa_{r}=0.1$ (top), $\kappa_{r}=0.3$ (middle) and $\kappa_{r}=0.5$ (bottom), with $\beta=0.1, n=1.0$, and $\eta=1.0$.

The numerical solution for rectangular waveguide is obtained by dividing the contour in a grid of $\mathrm{N}$ intervals. In order to decrease the size of the matrices, the $\mathrm{C}_{2}$ rotation symmetry can be considered and the problem is reduced to half of the transversal section. Furthermore, the $\mathrm{C}_{2}$ rotation symmetry allows to classify the eigenmodes in two groups, depending on the relation between the wavefields in the symmetric points, $\vec{E}^{ \pm}(\vec{r})=p \vec{E}^{ \pm}(-\vec{r})$, where $p= \pm 1$.

\section{RESULTS AND DISCUSSION}

The method has been applied to a X-band rectangular waveguide $(a=2.286 \mathrm{~cm}, b=1.016 \mathrm{~cm})$. Fig. 1 shows the dispersion curves for the first five modes as a function of the chirality parameter where the modes are labeled as $\mathrm{HE}_{x y}$ and $\mathrm{EH}_{x y}$, which correspond to $\mathrm{TE}_{x y}$ and $\mathrm{TM}_{x y}$, respectively, for

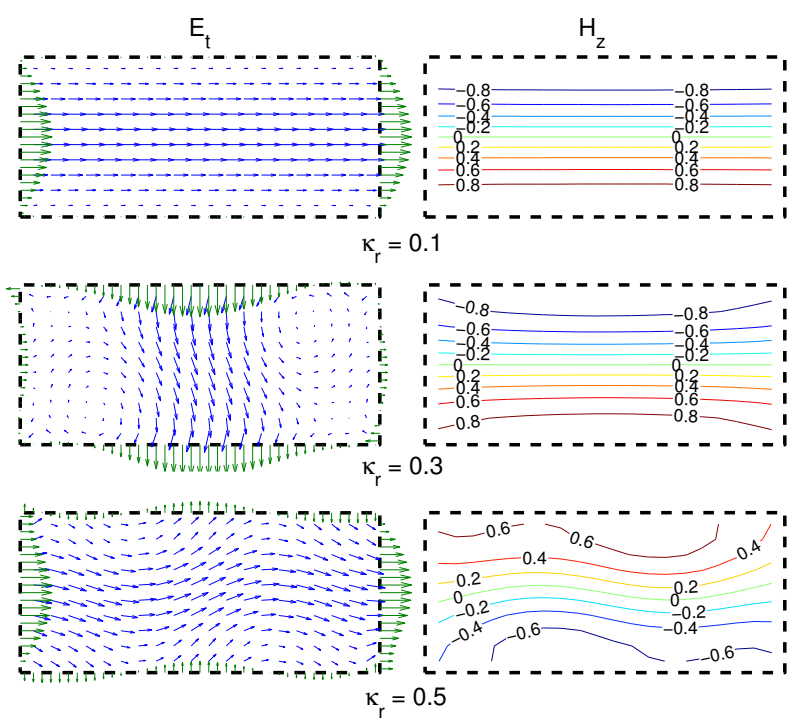

Fig. 3: Same as Fig. 2 for $\mathrm{HE}_{01}$ mode.
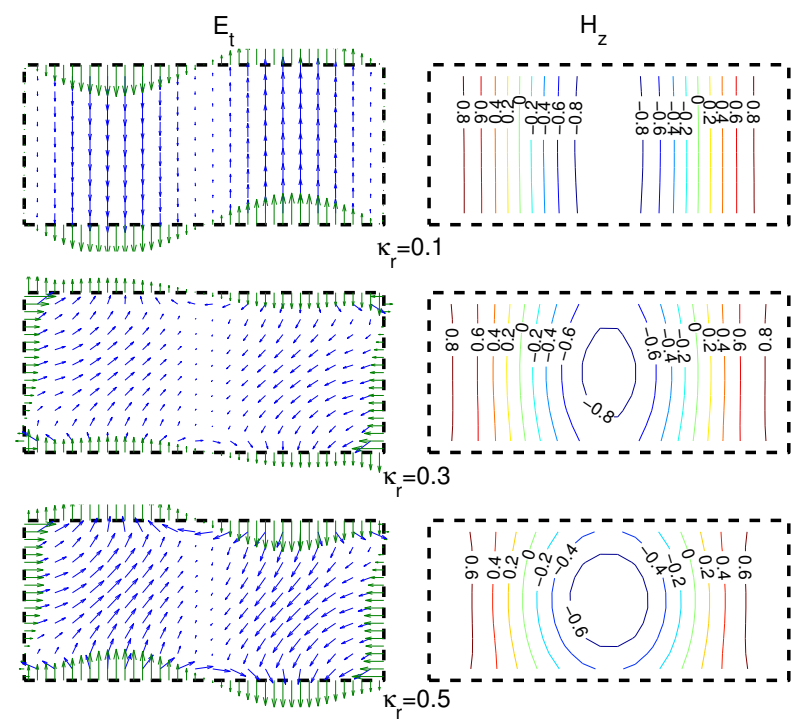

Fig. 4: Same as Fig. 2 for $\mathrm{EH}_{20}$ mode.

the limit case when $\kappa_{r}=0$. For a given frequency, the complex propagation constant was obtained as the one who makes null the determinant of the matrix. In some cases, this procedure can provide wrong values for $\beta$, that can be eliminated by adding extra points placed outside the section $S$ or from the field structure in the waveguide cross section.

Figures 2, 3, 4, 5 and 6 show vector and contour plots of $E_{t}$ and $H_{z}$, respectively, for the first five propagation modes and for three different values of $\kappa_{r}$. The chirality affects the electromagnetic field pattern by breaking the expected reflection symmetry of the dielectric case, which is replaced by a rotation symmetry. As it can be expected, neither TE nor TM pure modes are obtained in a rectangular chirowaveguide.

Fig. 2 shows the obtained results for the first propagation mode, $\mathrm{HE}_{10}$. For the low chirality case, $\kappa_{r}=0.1$, the fields 


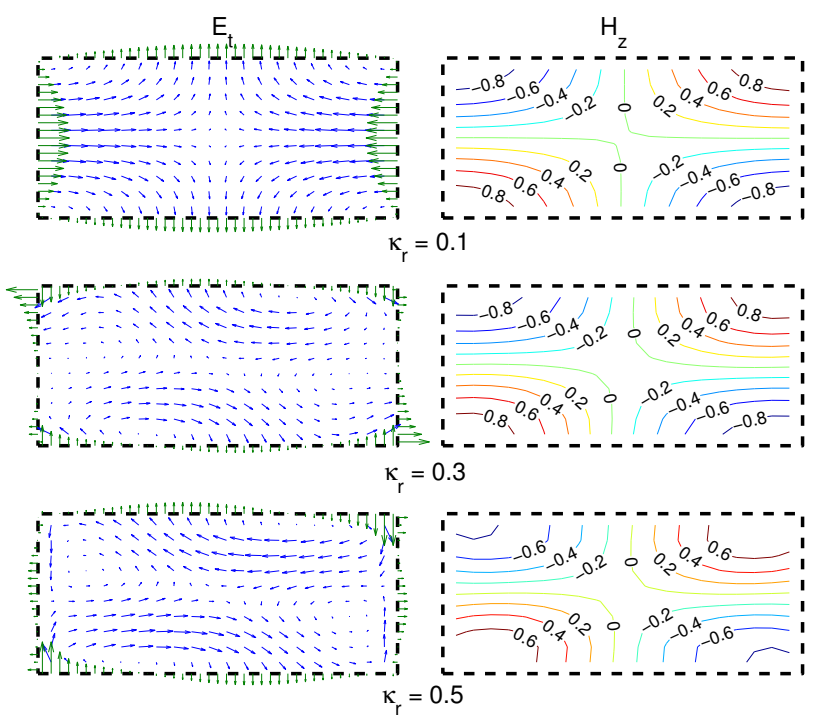

Fig. 5: Same as Fig. 2 for $\mathrm{HE}_{11}$ mode.

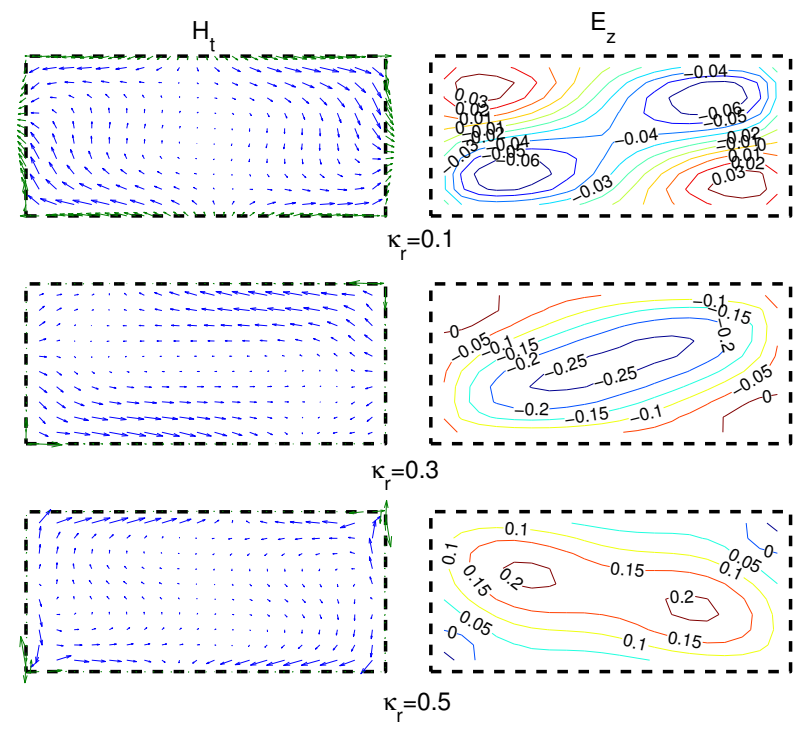

Fig. 6: Same as Fig. 2 for $\mathrm{EH}_{11}$ mode.

seem to keep the reflection symmetry, but this is not the case for the high chirality case, $\kappa_{r}=0.5$. As the chirality increases, the typical structure of the $\mathrm{TE}_{10}$ mode disappears. For example, the electric field is not always parallel to shortside of the waveguide $b$, but it points in the direction of the long-side, $a$. Furthermore, the electric field is not transversal to the propagation direction, $E_{z} \neq 0$, not shown in the figures. As we can expected, only the rotation symmetry is revealed for high values of chirality.

Fig. 3 shows the results for $\mathrm{HE}_{01}$ mode, where it can be observed that the typical $\mathrm{TE}_{01}$ structure disappears as the chirality increases. For example, the electric field is not parallel to the long side of the waveguide, $a$, as can be seen clearly at the center of the waveguide.

Fig. 4 and 5 show the obtained results for modes which correspond to $\mathrm{HE}_{20}$ and $\mathrm{HE}_{11}$, respectively. Again, for $\kappa_{r}=$ 0.1 the fields keep the expected patterns of the achiral case. As the chirality increases, the reflection symmetry disappears and the rotation symmetry revealed.

$\mathrm{TE}_{11}$ and $\mathrm{TM}_{11}$ are degenerated modes in the dielectric case and also the corresponding modes for the chirowaveguide, $\mathrm{HE}_{11}$ and $\mathrm{EH}_{11}$. For a given frequency, the excitation of a specific mode depends on the initial conditions, in our model the value for $E_{z}^{+}$or $\partial_{n} E_{z}^{+}$at the chosen contour point. Fig. 6 shows the results for the first EH mode, where it can be observed that the $H_{n} \neq 0$ at the PEC boundaries.

\section{COnclusions}

An application of the Green integral equations to uniform waveguides with arbitrary cross section filled with chiral media is presented in the paper. The model is first tested by solving the rectangular dielectric waveguide, and both the dispersion diagrams and the field structure of the dielectric waveguide are obtained. Then, the method is applied the chiral guide. The results show that chirality affects the electromagnetic field pattern by breaking the reflection symmetry of the TE modes and by producing a rotation symmetry in the field structure. The technique here presented can be adapted to most of the standard waveguide systems and also to the chiral fiber.

\section{ACKNOWLEDGMENT}

This work was supported by the Spanish Government (Research Projects TEC2014-55463-C3-1-P and TEC2014-55463C3-2-P) and by the European Commission (ERDF).

\section{REFERENCES}

[1] S. Zouhdi, A. Sihvola, and M. Arsalane (Eds.), Advances in Electromagnetics of Complex Media and Metamaterials, Nato Science Series II:, Vol. 89, Dordrecht, The Netherlandas: Kluver Academic Publisher, 2003.

[2] G. V. Eleftheriades, and K. G. Balmain, Negative-Refraction Metamaterials: Fundamental Principles and Applications Hoboken: USA, Wiley and Sons, 2005.

[3] Y. Hao, and R. Mittra, FDTD Modeling of Metamaterials Theory and Applications Noorwood, USA: Artech House Inc, 2009.

[4] N. Engheta, and P. Pelet, "Modes in chirowaveguides", Optics Lett., Vol. 14, No. 11, pp 593-595, June 1989.

[5] L. W. Li, M. S. Leong, P. S. Kooi, T. S. Yeo, and K. H. Tan, "Rectangular modes and dyadic Green's functions in a rectangular chirowaveguide. I. Theory", IEEE Trans. Microw. Theory Techn., vol. 47, pp 67-73, 1999.

[6] A. Gómez, A. Lakhtakia, J. Margineda, G. J. Molina-Cuberos, M. J. Núñez, J. A. Saiz Ipina, A. Vegas, and M. A. Solano, "Full-Wave Hybrid Technique for 3-D Isotropic-Chiral-Material Discontinuities in Rectangular Waveguides: Theory and Experiment", IEEE Trans. Microw. Theory Techn., vol. 56, pp 2815-2825, 2008.

[7] A. L. Topa, C. R. Paiva, and A. M. Barbosa, "Electromagnetic wave propagation in chiral h-guides", Prog. Electromagnetic Res., vol. 103, pp 285-303, 2010.

[8] J. B. Pendry, "A Chiral Route to Negative Refraction”, Science, Vol. 306, pp 1353-1355, 2004

[9] M. Sancho, "Integral formulation of the boundary value problem in waveguides", Am. J. PHys., vol. 48, pp 1083-1087, 1980.

[10] I. V. Lindell, A. H. Sihvola, S. A. Tretyakov, and A. J. Vitanen, Electromagnetic Waves in Chiral Media, Boston, USA: Artech House, 1994. 